\title{
Usabilidade de aplicativos móveis educacionais infantis: design e avaliação de interação do Fantastic Pirates
}

\author{
Caio Almeida Abreu - Escola Politécnica/UFBA - caioabreu@ outlook.com \\ Jean Clemisson Santos Rosa - IME/UFBA - jean.rosa@ ufba.br Ecivaldo de \\ Souza Matos - IME/UFBA - ecivaldo@ufba.br
}

\begin{abstract}
Resumo: O mercado de aplicativos móveis educacionais apresenta uma gama diversificada de opções destinadas à satisfação de diversos públicos, sobretudo o infantil. Parte considerável desses aplicativos são jogos e abrange diversas idades, mas em alguns casos desconsideram características intrínsecas à educação infantil, em que crianças têm necessidades de interações diferenciadas e necessidades educacionais específicas. Nesse sentido, este artigo apresenta resultados da aplicação de um conjunto de heurísticas desenvolvidas especificamente para promoção da usabilidade técnica e pedagógica de aplicativos móveis educacionais infantis. Essas heurísticas foram utilizadas para orientar o design e a avaliação de interação do jogo educativo Fantastic Pirates. A avaliação foi realizada por meio de questionários e testes de usabilidade, usando think-aloud com crianças de até 10 anos. A aderência desse conjunto de heurísticas ao contexto infantil foi avaliada e constatada, bem como o grau de satisfação dessas crianças em relação à usabilidade do jogo, considerando o conjunto de heurísticas desenvolvido.
\end{abstract}

Palavras-chave: usabilidade; usabilidade pedagógica; jogos educativos; interação criança-computador.

\section{Usability of educational mobile app for children: interaction design and evaluation of Fantastic Pirates}

\begin{abstract}
The market for educational mobile applications presents a diverse range of options aimed at the satisfaction of different audiences, especially children. A considerable portion of these applications are games and cover multiple ages, but in some cases disregard features intrinsic to childhood education where children have differentiated interactions needs and specific educational needs. In this sense, this paper presents results of the application of a set of heuristics specifically developed to promote the technical and pedagogical usability of mobile educational applications for children. These heuristics were used to guide the interaction design and evaluation of the educational game Fantastic Pirates. The adherence of this heuristics' set to the child context was evaluated and checked, as well as the level of satisfaction of these children in relation to the game usability, considering the set of heuristics developed.
\end{abstract}

Keywords: usability; pedagogical usability; educational games; child-computer interaction.

\section{Introdução}

A utilização de aplicativos móveis educacionais tem crescido e aberto espaço para o mobile learning, termo utilizado nos estudos de investigação que relaciona 
mobilidade, aprendizagem e tecnologias móveis (Silva e Gomes, 2015). Segundo Winckler e Pimenta (2002), a escolha de software de apoio ao ensino e à aprendizagem não deve se pautar por metodologias para uma população alvo genérica; para aprendizagens apenas individuais, controle dos resultados da avaliação, dentre outros, pois não serão precisos, devido a ausência do contexto e de outras variáveis, como tipo de aplicação em questão e perfil dos usuários.

Além dos fatores pedagógicos mencionados, fatores técnicos, como a usabilidade, devem ser considerados durante a concepção de softwares educacionais. A usabilidade, enquanto categoria de qualidade de interação humano-computador, é indispensável a softwares dessa natureza (Rosa, Schwarzelmüller e Matos, 2015).

De acordo com Nielsen (2012), o conceito de usabilidade está relacionado intrinsecamente aos seguintes atributos: satisfação do usuário, facilidade e memorização da utilização do software, tratamento de erros e a eficiência no uso software. Nesse contexto, Nielsen apresentou 10 heurísticas de usabilidade e indicou a necessidade de adaptações nas heurísticas propostas para atender sistemas de domínios diferentes. Desse modo, nota-se que a preocupação com a usabilidade deve considerar os diferentes domínios de softwares e diferentes perfis de usuários.

Há diversas heurísticas de usabilidade para avaliação de softwares (Knoll, 2012; Salazar et al., 2012; Almeida et al., 2013). Todavia, D’Carlo, Barbosa e Oliveira (2016) apontaram a insuficiência de heurísticas que abordassem aspectos específicos de usabilidade para aplicativos móveis educacionais. A partir dessa evidência, e da evidência de que crianças têm necessidades de interação diferenciadas e necessidades educacionais específicas (An et al., 2013), Abreu (2017) propôs um conjunto de heurísticas específicas para avaliação de usabilidade de aplicativos móveis educacionais.

Baseado nessas heurísticas de usabilidade específicas, foi desenvolvido um aplicativo móvel educacional de uso infantil, o Fantastic Pirates (FP), destinado a crianças de até 10 anos de idade (Abreu, Rosa e Matos, 2017a). Posteriormente, outro aplicativo, o Fantastic Pirates Educator (FPE), destinado aos educadores (professores e pais), foi desenvolvido para dar suporte ao FP. O FPE não será detalhado neste artigo por estar fora do escopo da pesquisa apresentada.

Este artigo apresenta os resultados da aplicação de um conjunto de heurísticas desenvolvidas especificamente para promoção da usabilidade técnica e pedagógica de aplicativos móveis educacionais infantis. Essas heurísticas foram utilizadas para orientar o design e a avaliação de interação do jogo educativo FP. Avaliou-se a satisfação das crianças em relação à usabilidade do jogo e a aderência das heurísticas ao contexto de aplicativos móveis educacionais infantis.

O texto está organizado em seis seções. A Seção 2 apresenta o referencial teórico e os trabalhos relacionados sobre heurísticas de usabilidade para aplicativos móveis educacionais e heurísticas de usabilidade específicas para aplicativos móveis educacionais infantis. A metodologia de pesquisa será apresentada na terceira seção. As 
Seções 4 e 5 apresentam a análise dos resultados da avaliação do aplicativo FP, seguidas das conclusões e trabalhos futuros, respectivamente.

\section{Heurísticas de usabilidade para aplicativos móveis educacionais}

D'Carlo, Barbosa e Oliveira (2016) evidenciaram a insuficiência de heurísticas que explorem aspectos específicos de usabilidade para aplicativos móveis educacionais. Adicionalmente, elas propuseram um conjunto de heurísticas para avaliar a usabilidade de aplicativos dessa natureza.

Para a criação desse conjunto, as autoras consideraram três perspectivas: (1) heurísticas genéricas para aplicativos móveis, por descreverem requisitos básicos de usabilidade para a interface de qualquer tipo de aplicativo; (2) requisitos de usabilidade de sistemas educacionais apresentados por Nokelainen (2005) e Silveira e Carneiro (2012); e (3) o conhecimento de um especialista em educação. Posteriormente, essas heurísticas passaram por avaliações com especialistas em Educação e Interação Humano-Computador, com intuito de compreender sua relevância no contexto educacional (D’Carlo, Barbosa e Oliveira, 2016). O conjunto resultante é apresentado na Quadro 1.

\begin{tabular}{|l|l|}
\hline Cód. & \multicolumn{1}{|c|}{ Nome } \\
\hline HG1 & Consistência e bom aproveitamento do espaço da tela \\
\hline HG2 & Disponibilidade (Acesso de qualquer lugar) e Portabilidade \\
\hline HG3 & Facilidade de acesso às funcionalidades \\
\hline HG4 & Resposta Imediata e Visibilidade \\
\hline HG5 & Adequação de mensagem à funcionalidade e ao usuário \\
\hline HG6 & Prevenção de erros e recuperação rápida ao último estado estável \\
\hline HG7 & Aprendizado orientado a um objetivo \\
\hline HG8 & Aprendizagem e Recordação no processo de Interação \\
\hline HG9 & Controle de aprendizado e Flexibilidade \\
\hline HG10 & Disponibilizar diferentes experimentações de aprendizado, incluindo erros \\
\hline HG11 & Aprendizado cooperativo/colaborativo \\
\hline HG12 & Motivação \\
\hline HG13 & Consolidação de novos aprendizados (Aplicabilidade) \\
\hline HG14 & Ajuda e documentação \\
\hline
\end{tabular}

Quadro 1 - Heurísticas específicas para usabilidade de aplicativos educacionais Fonte: D'Carlo, Barbosa e Oliveira (2016)

\subsection{Heurísticas específicas para usabilidade de aplicativos educacionais infantis}

Ainda que relevantes, as heurísticas propostas por D'Carlo, Barbosa e Oliveira (2016) não consideram especificidades do domínio infantil. Nesse sentido, Abreu (2017) propôs um conjunto de heurísticas de usabilidade específicas para aplicativos móveis educacionais infantis, apresentado no Quadro 2. O autor considerou as heurísticas para aplicativos móveis educacionais, propostas por D'Carlo, Barbosa e Oliveira (2016), e elementos caracterizadores do domínio infantil segundo An et al. (2013) e Gomes et al. (2015).

Essas heurísticas foram concebidas por meio de (i) análise crítica das heurísticas de D'Carlo, Barbosa e Oliveira (2016); (ii) harmonização para eliminação de elementos semelhantes ou repetidos; e (iii) avaliação por especialistas de Informática na Educação, 
de Interação Humano-Computador e por profissionais da Educação Infantil (Abreu, 2017). Para realizar a análise crítica das heurísticas apresentadas por D'Carlo, Barbosa e Oliveira (2016) foi observado estudos das áreas de Educação Infantil e Psicologia da Educação, tais como Kishimoto (1994) e Vygotsky (2004). Os autores também realizaram um estudo experimental com a finalidade de comparar as heurísticas apresentadas no Quadro 1 (genéricas) com as heurísticas apresentadas nesta seção (específicas do domínio infantil). Esse processo não será apresentado em detalhes, mas pode ser consultado em Abreu, Rosa e Matos (2017b).

\begin{tabular}{|l|l|}
\hline Cód. & \multicolumn{1}{|c|}{ Nome } \\
\hline HE1 & Facilidade de Uso \\
\hline HE2 & Design da Tela \\
\hline HE3 & Apresentação da Informação \\
\hline HE4 & Afetividade \\
\hline HE5 & Motivação \\
\hline HE6 & Aprendizado Orientado a Metas e Objetivos \\
\hline HE7 & Papel do Instrutor \\
\hline HE8 & Controle do Aluno \\
\hline HE9 & Aprendizado Cooperativo \\
\hline HE10 & Linguagem alvo \\
\hline HE11 & Entretenimento \\
\hline HE12 & Uso do Lúdico \\
\hline HE13 & Criatividade e Imaginação \\
\hline HE14 & Esforço Cognitivo \\
\hline HE15 & Sentido Lógico \\
\hline HE16 & Coerência no Conteúdo \\
\hline HE17 & Feedback \\
\hline HE18 & Disponibilidade e Portabilidade \\
\hline HE19 & Resposta Imediata e Visibilidade \\
\hline HE20 & Adequação de Mensagem à Funcionalidade e ao Usuário \\
\hline HE21 & Prevenção de Erros e Recuperação Rápida do Sistema \\
\hline HE22 & Aprendizagem e Recordação \\
\hline HE23 & Diferentes Meios de Aprendizado \\
\hline
\end{tabular}

Quadro 2 - Heurísticas de usabilidade específicas para aplicativos móveis educacionais infantis Fonte: Abreu (2017)

\section{Metodologia}

Para avaliar o aplicativo Fantastic Pirates $^{1}$, desenvolvido para a plataforma Android $^{\circledR}$, foi utilizado o método de avaliação de usabilidade com think-aloud (Markopoulos et al., 2008). Esse método incluiu gravações de áudio, transcrições e gravações da manipulação das telas do jogo. Também foi utilizado um questionário com base em 14 (HE1, HE2, HE3, HE4, HE5, HE6, HE8, HE10, HE13, HE14, HE16, HE17, $H E 10, H E 22)$ das 23 heurísticas destinadas a aplicativos móveis educacionais infantis.

Desse modo, nove heurísticas não foram diretamente avaliadas pelo questionário. Porém, todas as heurísticas foram avaliadas indiretamente pelo método de think-aloud. Isso se deve ao fato de que essas nove heurísticas são muito específicas e algumas delas não se aplicam à avaliação pelas crianças, mas pelos educadores. Esse foi o caso das heurísticas HE7 (Papel do Instrutor), HE9 (Aprendizado Cooperativo), HE11

\footnotetext{
${ }^{1}$ Mais detalhes sobre o FP podem ser encontrados em Abreu, Rosa e Matos (2017a).
} 
(Entretenimento), HE12 (Uso do Lúdico), HE15 (Sentido Lógico), HE18 (Disponibilidade e Portabilidade), HE19 (Resposta Imediata e Visibilidade), HE21 (Prevenção de Erros e Recuperação Rápida do Sistema) e HE23 (Diferentes Meios de Aprendizado).

A fim de averiguar a consistência do questionário, bem como a execução da avaliação por meio do think-aloud (gravação de tela e áudio) ao contexto infantil, foram realizados dois pré-testes com crianças de 6 e 10 anos de idade. Após executar os pré-testes e realizar os ajustes necessários, a avaliação qualitativa do aplicativo FP foi conduzida com cinco crianças nesse mesmo intervalo de idade. As crianças que participaram da pesquisa foram selecionadas por conveniência. $O$ número de participantes corresponde à indicação média de avaliação qualitativa de usabilidade com participação de sujeitos.

Vale salientar que as heurísticas de usabilidade para aplicativos móveis educacionais infantis considera crianças de até 10 anos de idade. No entanto, como o aplicativo desenvolvido com base nas heurísticas requer algum contato das crianças com o processo de alfabetização, a avaliação foi conduzida com crianças entre 6 e 10 anos de idade (também dentro do intervalo de abrangência das heurísticas), visto que o processo de alfabetização no Brasil se inicia entre 5 e 6 anos de idade.

Nas avaliações foram utilizados dois smartphones: o Motorola Nexus 6 e o Samsung Galaxy J7; ambos com características semelhantes, requisito importante para diminuir os efeitos da diferença de hardware no processo avaliativo. Os aplicativos foram previamente executados nesses smartphones com intuito de evitar riscos e limitações que poderiam surgir na condução da avaliação com crianças. Seus responsáveis assinaram um termo de consentimento livre e esclarecido (TCLE) permitindo a participação anônima dessas crianças.

As crianças inicialmente receberam o smartphone e foi solicitado a elas que executassem o aplicativo FP e jogassem à vontade. Previamente, aplicativos de gravação de tela e áudio foram colocados em execução nos smartphones fornecidos às crianças. Ao final da interação, as perguntas do questionário foram conduzidas pelos pesquisadores e as crianças puderam respondê-las e tecer observações sobre o aplicativo.

Na próxima seção serão apresentados e discutidos os resultados da avaliação considerando o questionário e a avaliação de usabilidade com think-aloud. Em seguida, são apresentadas as conclusões deste artigo.

\section{Resultados}

Os resultados obtidos por meio do questionário com crianças, utilizado para avaliar o grau de satisfação das crianças em relação à usabilidade do aplicativo FP, indicaram que o aplicativo teve um percentual de satisfação pelas crianças de $84 \%$ considerando as heurísticas propostas $(\mathrm{Hn})$. Nenhuma heurística teve grau de satisfação menor que $20 \%$; e $64 \%$ das heurísticas foram qualificadas com grau de satisfação máximo (100\%) atribuído por todas as crianças. A Figura 1 apresenta os percentuais de satisfação de cada heurística em relação à usabilidade do aplicativo FP, conforme indicado no questionário.

A análise dos dados coletados por meio do questionário foi apoiada pelo método de think-aloud, que resultou em mais dados coletados por meio de gravações da manipulação das telas do jogo e do áudio ambiente (sons e expressões emitidas pelas 
crianças). Após a análise das transcrições das falas das crianças durante o processo avaliativo, foi possível destacar algumas considerações.

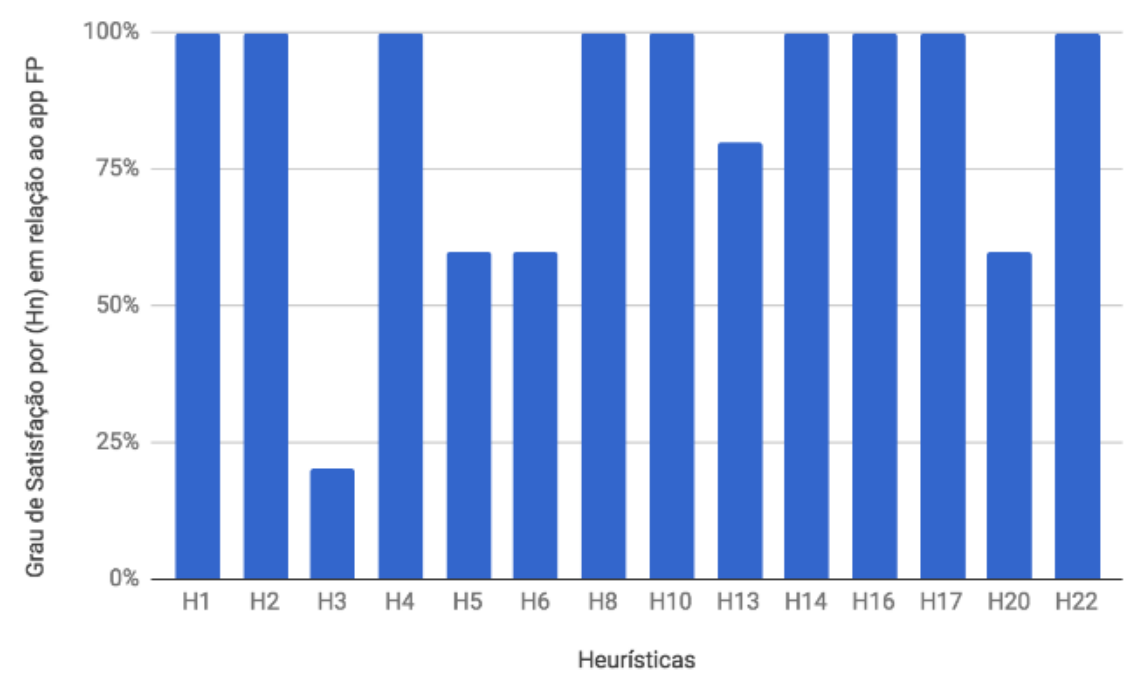

Figura 1 - Resultado das respostas do questionário. Fonte: Abreu (2017)

A Heurística 5 (Motivação) foi encontrada por meio de falas como: "Ganhei na primeira vez esse tanto de ouro!', "Ganhei!’. O ouro mencionado pelas crianças foi um dos mecanismos de pontuação para motivação da criança. A recompensa por meio de prêmios foi utilizada durante todo o jogo e distribuída em subjogos internos, como tesouros que são convertidos em moedas de ouro, agregando valor à pontuação.

A Heurística 4 (Afetividade) foi evidenciada por meio de falas como "Jogo massa!". A ênfase na fala da criança, juntamente à expressão "massa", revela que os elementos conectados ao mundo infantil presentes no jogo culminaram na criação de laços emocionais entre o usuário e o jogo. Todas as crianças que participaram da avaliação expressaram se sentirem satisfeitas no aspecto afetivo ( $c f$. Figura 1).

Apesar de a maioria das crianças estarem em processo de alfabetização, a Heurística 10 (Linguagem Alvo) se mostrou consistente tanto pela análise dos dados sumarizados na Figura 1, quanto pela escassez de questionamentos sobre o significado das palavras utilizadas no jogo.

Os resultados obtidos por meio das gravações das telas mostraram que algumas crianças tiveram dificuldades na manipulação da tela referente ao subjogo "Roleta Mágica", Figura 2 (lado esquerdo). Essas crianças tocaram diretamente na roleta (ao centro da tela) ou fizeram movimentos circulares nela na expectativa de que a roleta começasse a girar. Isso provavelmente se deve ao fato de a roleta estar localizada no centro da tela (primeiro ponto de foco por parte do usuário) e suas múltiplas cores atraírem a atenção da criança. Concomitantemente, o botão que deveria ser utilizado para fazer a roleta girar acaba sendo projetado na visão periférica da criança. Acredita-se que pelas limitações da visão periférica humana, algumas crianças demoraram alguns segundos para perceber a presença do botão e usar o software conforme esperado pelos pesquisadores. Outra hipótese sobre a roleta é que as crianças estão mais familiarizadas com a interação direta com os objetos da interface e, portanto, elas ignoraram o botão e clicaram diretamente na roleta.

A tela de descoberta do baú secreto, Figura 2 (lado direito), apresentada no jogo a cada ciclo de cinco questões, também gerou dificuldade para a maioria das crianças. 
Apesar de haver um botão de ajuda (representado pelo símbolo de interrogação “?”) com função de revelar todas as dicas acumuladas no jogo para caso de a criança não se recordar, parte considerável das crianças pediram ajuda diretamente aos pesquisadores. Provavelmente isso ocorreu devido a dificuldade de leitura por parte das crianças, ainda em processo de alfabetização; assim, parte algumas delas não entenderam o propósito das dicas no jogo e, ao atingir a tela que perguntava o objeto referente às dicas, essas crianças solicitaram ajuda.
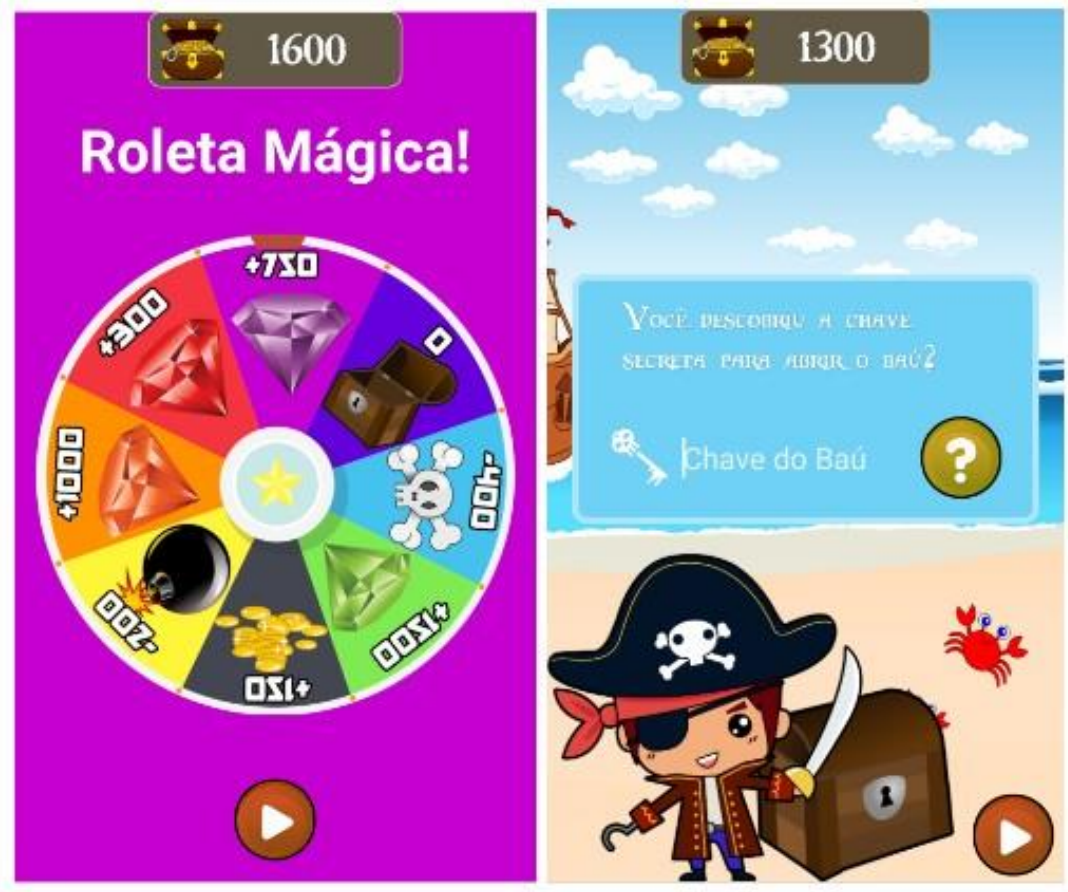

Figura 2 - Tela do subjogo "Roleta Mágica"(à esquerda) e Tela de adivinhação do "Baú Secreto" (à direita)

Ambas as dificuldades apresentadas nas telas representadas na Figura 2 ajudaram a compreender o único nível de satisfação inferior a $60 \%$, atribuído à Heurística 3 (Apresentação da Informação), visto que essa heurística trata da facilidade de encontrar as informações básicas do aplicativo como ajuda, configurações, próximas ações, objetivos e documentação. Como a maioria das crianças teve dificuldade em alguma das telas citadas, a satisfação atribuída à Heurística 3 foi prejudicada.

Uma das crianças apontou o nível de dificuldade das perguntas do jogo ao relatar: "O jogo foi legal, mas teve perguntas difíceis, tipo eu acertei umas duas e errei três. Eu pensei que ia ser as perguntas fáceis, mais bestas, mas foi pergunta difícil. Eu fiquei surpreso na hora!'. O nível de dificuldade do jogo é personalizável e reflete o poder da Heurística 7 (Papel do Instrutor), que apesar de não ter sido avaliada por meio do questionário, ela se fez presente no aplicativo e foi avaliada por meio do think-aloud.

Uma das crianças indicou um dos desafios tecnológicos do aplicativo, ao afirmar que "A única coisa que eu não gostei do jogo foi que demorava muito para carregar", referindo-se ao momento em que necessita de acesso à internet para acessar o jogo. Essa situação ocorreu devido ao fato de no momento do teste, o sinal de internet (wi-fi) ter sido perdido; portanto, o smartphone automaticamente ativou o uso do plano de dados 
(tecnologia 4G), com recursos limitados de conexão. O FP faz uso da internet apenas no início do jogo, para fazer download do conteúdo (perguntas, dicas e demais configurações), e ao final do jogo, para enviar um relatório completo de erros e estatísticas sobre o desempenho da criança para o aplicativo de suporte aos educadores, o FPE.

Outros problemas de interação de menor grau foram evidenciados pelas crianças. Uma criança teve dificuldade para encontrar as letras no teclado do smartphone, como revela a fala "onde é o U?". Algumas crianças acertaram a adivinhação correspondente à chave para abrir o baú secreto, que era a palavra "anel", mas demonstraram dúvidas na escrita da palavra ao questionarem "anel é com o quê?", "é com L, né?". Outra digitou "ameo". Esses problemas foram evidenciados conforme a escala de idade das crianças, com impacto maior sobre aquelas de menor idade, tais como seis ou sete anos, ainda em processo de alfabetização.

Desse modo, parte considerável dos problemas encontrados refletem a dificuldade de leitura e escrita do conteúdo. Contudo, esses problemas não refletem necessariamente dificuldade de interação, visto que o conteúdo do aplicativo é regulado pelo papel do instrutor, que pode ajustar a dificuldade do jogo de acordo com o que insere no jogo, conforme a idade e as necessidades educacionais da criança.

\section{Considerações Finais}

Por meio da análise dos dados, verificou-se que o aplicativo satisfez as crianças de acordo com o que fora estabelecido a priori, considerando as especificidades do público alvo, uma vez que o nível de satisfação do público infantil (medido pelo questionário) em relação à usabilidade do aplicativo FP foi de $84 \%$. Além disso, infere-se que, como o aplicativo foi desenvolvido baseado nas heurísticas de usabilidade específicas para aplicativos móveis educacionais infantis, essas heurísticas também se mostraram adequadas ao domínio infantil. De qualquer modo, mais estudos com grupos de crianças e educadores são necessários para identificarmos com clareza a abrangência e os limites de avaliação dessas heurísticas.

Do ponto de vista científico e tecnológico, o conjunto de heurísticas poderá ser utilizado para avaliar a usabilidade de aplicativos móveis educacionais, considerando características intrínsecas ao público infantil. Esse conjunto de heurísticas poderá ser explorado para avaliar aplicativos tanto em fase de projeto quanto aqueles já desenvolvidos. Na fase de projeto, essas heurísticas podem ser utilizadas para o design de interação, de modo a melhorar a qualidade da interação do aplicativo. Ao avaliar aplicativos já desenvolvidos, as heurísticas podem ser úteis ao redesign de interação e/ou para desenvolvimento de estratégias de marketing para evidenciar a adequação do aplicativo ao público infantil.

Acredita-se no potencial de benefício social desse trabalho, uma vez que os aplicativos FP e FPE poderão ser utilizados por educadores (professores e pais) para estimular crianças nos a aprender de forma interativa, lúdica e divertida.

Como trabalhos futuros, espera-se fazer o redesign de interação do aplicativo FP, levando em consideração os dados coletados e, posteriormente, conduzir novas avaliações com crianças para que o aplicativo atinja um nível de satisfação em relação à sua usabilidade superior ao atual (84\%). Bem como desenvolver novos estudos para definição clara dos limites metodológicos desse conjunto de heurísticas. 


\section{Agradecimentos}

Os autores agradecem o apoio do Grupo de Pesquisa e Extensão em Informática, Educação e Sociedade - Onda Digital; à Coordenação de Aperfeiçoamento de Pessoal de Nível Superior (Capes) pelo financiamento parcial desta pesquisa; e às crianças e responsáveis que participaram desta pesquisa.

\section{Referências}

ABREU, C.A.; ROSA, J.C.S.; MATOS, E. Fantastic Pirates: software de apoio ao ensino e à aprendizagem infantil. In: Anais dos Workshops do Congresso Brasileiro de Informática na Educação, 2017. Recife. Anais. Porto Alegre: Sociedade Brasileira de Computação, 2017a. p. 252-259.

ABREU, C.A.; ROSA, J.C.S.; MATOS, E. Aplicabilidade de Heurísticas de Usabilidade para Aplicativos Móveis Educacionais Infantis. In: Brazilian Symposium on Human Factors in Computing Systems, 2017. Joinville. Anais. Porto Alegre: Sociedade Brasileira de Computação, 2017b. p. 70-71.

ABREU, C.A. Heurísticas de Usabilidade para Aplicativos Móveis Educacionais Infantis. Salvador: POLI/UFBA, 2017. 82. Monografia.

ALMEIDA, R.X.E.; FERREIRA, S.B.L.; SILVEIRA, D.S.S.; PIMENTAL, M. GOLDBACH, R.; BESSA, A.T. Heurísticas de Usabilidade Orientadas às Redes Sociais. In: Encontro de Administração da Informação, 4., 2013. Bento Gonçalves. Anais. Rio de Janeiro: ANPAD, 2013. p. 1-15.

AN, D.Y.; SILVA C.D.; RIBEIRO D.G.G.; ROCHA P.B.R.; MALTINTI C.; NUNES V.B.; FÁVERO R. "Digita - Um jogo educativo de apoio ao processo de alfabetização infantil”. In: Simpósio Brasileiro de Informática na Educação, 24., 2013. Campinas. Anais. Porto Alegre: Sociedade Brasileira de Computação, 2013, p. 154-163.

D'CARLO, D.; BARBOSA, G.A.R.; OLIVEIRA, E.R. Usabilidade em aplicativos móveis educacionais: Um conjunto de heurísticas para avaliação. In: Simpósio Brasileiro de Informática na Educação, 27., 2016. Uberlândia. Anais. Porto Alegre: Sociedade Brasileira de Computação, 2016, p. 777-786.

GOMES, T.C.S.; BARRETO P.P.; LIMA I.R.A.; FALCÃO T.P. Avaliação de um jogo educativo para o desenvolvimento do pensamento computacional na educação infantil. In: Workshops do Congresso Brasileiro de Informática na Educação, 6., 2015. Maceió. Anais. Porto Alegre: Sociedade Brasileira de Computação, 2015, p. 1349-1358.

KISHIMOTO, T.M. O jogo e a educação infantil. Perspectiva. Florianópolis, 1994.

KNOLL, R.C. Desenvolvimento de heurísticas de usabilidade para tablets. Caderno de Estudos Tecnológicos. Bauru, 2., p 1-75, 2012.

MARKOPOULOS, P.; READ, J.C.; MACFARLANE, S.; HÖYNIEMI, J. Evaluating Children's Interactive Products: Principles and Practices for Interaction Designers. Morgan Kaufmann Publishers Inc., San Francisco, CA, EUA. 2008. 
NIELSEN, J. Usability 101: Introduction to Usability. NN/g Nielsen Norman Group, 2012. Disponível em: <https://www.nngroup.com/articles/usability-101-introduction-to-usability/>. Acesso em: 01 de março de 2017.

NOKELAINEN, P. The technical and pedagogical usability criteria for digital learning material. In: World Conference on Educational Multimedia, Hypermedia and Telecommunications, 9., Waynesville. Anais. Waynesville: Association for the Advancement of Computing in Education, 2005. p. 1011-1016.

ROSA, J.C.S.; SCHWARZELMÜLLER, A.F.; MATOS, E. Avaliação heurística da rede social educacional TecCiencia. In: Escola Regional de Computação Bahia - Alagoas Sergipe, 2015. Salvador. Anais. Porto Alegre: Sociedade Brasileira de Computação, 2015. s/p.

SALAZAR, L.H.A.; LACERDA, T.; WANGENHEIM, C.G.; BARBALHO, R. A. Customizando heurísticas de usabilidade para celulares. In: Brazilian Symposium on Human Factors in Computing Systems, 11., 2012. Cuiabá. Anais. Porto Alegre: Sociedade Brasileira de Computação, 2012. p. 37-38.

SILVA, V.G.; GOMES, M.J. Dos dispositivos móveis à aprendizagem ubíqua - Da usabilidade técnica à usabilidade pedagógica. Revista de Estudios e Investigación en Psicología y Educación. v. extr., n. 13, p. 211-214, 2015.

SILVEIRA, M.S.; CARNEIRO, M.L.F. Diretrizes para Avaliação da Usabilidade de Objetos de Aprendizagem. In: Simpósio Brasileiro de Informática na Educação, 23., 2012. Rio de Janeiro. Anais. Porto Alegre: Sociedade Brasileira de Computação, 2012, $\mathrm{s} / \mathrm{p}$.

VYGOTSKY, L.S. Imagination and Creativity in Childhood. Journal of Russian and East European Psychology, v. 42, n. 1, p. 7-97, 2004.

WINCKLER, M.A.A.; PIMENTA, M. S. Avaliação de Usabilidade de sites Web. In: NEDEL, L. (Org.) X Escola de Informática da SBC-Sul, Porto Alegre: Sociedade Brasileira de Computação, 2002, p. 85-137. 